\title{
Tunable Fano resonances in a robust quasi- cylindrical microresonator photonic system
}

\author{
Xueying Jin, Mengyu Wang, Yongchao Dong, Liming Chen, Fei Li, and Keyi Wang* \\ Department of Precision Machinery and Precision Instrumentation, University of Science and \\ Technology of China, Hefei, Anhui, 230026 China
}

\begin{abstract}
The control of Fano resonances is of critical importance to opto-electronic and all-optical switching devices, light delay and storage, high sensitivity sensors, and quantum information processors. In this paper, we experimentally and theoretically demonstrate that controllable electromagnetically induced transparency (EIT)-like and Fano resonances can be achieved in a single quasi-cylindrical microresonator (QCMR). Robust and selective excitation of localized axial modes in a high quality QCMR is firstly demonstrated. Based on this stable platform, EIT-like lineshapes can be tuned and converted into Fano resonances by vertically moving the resonator. Moreover, by horizontally scanning the resonator, the transmission spectrum exhibits periodically changed Fano-like lineshapes. It is reported for the first time that the above two kinds of Fano resonances originated from different mechanisms can work on the same mode simultaneously. Our approach, demonstrated in this work, provides a robust photonic platform for accessing, controlling, and engineering the Fano resonances.
\end{abstract}

\section{Introduction}

Fano resonances, sometimes manifesting as electromagnetically induced transparency (EIT), comes from quantum interference of a discrete excited state of an atom and a continuum, which was firstly discovered by Ugo Fano [1]. Fano resonance exhibits an asymmetric lineshape with sharp slope, while EIT shows a beautiful transparent window through eliminating the resonant adsorption. The magnitude and phase of the field change dramatically at Fano resonances. Consequently, a small perturbation in the system results in very large change in magnitude and phase. This property holds great potential in fruitful applications, including but not limited to opto-electronic devices, high-sensitivity sensors [2], optical storage [3-4], slow light [5], narrowband filtering, and nonlinearity enhancement.

Previously, Fano resonances have been demonstrated in coupled whispering gallery mode (WGM) microcavities [6-8], a multimode tapered fiber coupled a microsphere [9], or in a single WGMR [10-14]. To achieve this, a high quality (Q) mode and a low Q mode have to be excited simultaneously in a narrow frequency range, and can be tuned independently. Various tuning approaches have been developed, such as temperature tuning

\footnotetext{
* Corresponding author: kywang@ustc.edu.cn
} 
[15], optical gain tuning [11], pressure tuning [14], stretching tuning [13], and coupling gap tuning [12]. In this paper, we study dynamic controlled Fano resonances in a quasicylindrical microresonator (QCMR). Here, we take a different way called "displacement tuning”. Specifically, in Sec. 2, we first construct a robust QCMR-fiber coupling platform and report selective excitation of high Q localized axial modes. In Sec. 3, by vertically moving the QCMR along its axis, the EIT and Fano resonances are generated and dynamically controlled. This originates from destructive interference between a high $\mathrm{Q}$ and a low Q mode. They can convert into each other by adjusting the coupling condition. In addition, by horizontally moving the QCMR, another kind of periodically changed Fano resonances are exhibited and engineered, which can be attributed to multimode coupling and modal dispersion in the fiber. The most important point is that the two different mechanisms that induce Fano resonances can work on the same mode simultaneously. Sec. 4 provides a summary of our work.

\section{Coupling platform construction and mode selective excitation}

In this section, we first fabricate a high Q QCMR and then construct a robust coupling platform. Next, we demonstrate selective excitation of localized axial modes in the QCMRmicrofiber system. We analyze the spatial and spectral field distribution of QCMR experimentally and numerically and find that they agree well with each other. Our QCMR exhibits clean spectra, a high Q up to $3.1 \times 10^{7}$, a favorable frequency spacing $\sim 3.91 \mathrm{GHz}$, and highly non-degenerated localized axial modes. These highly non-degenerated WGMs are well separated along the axis of the resonator and provide good mode-selection capability.

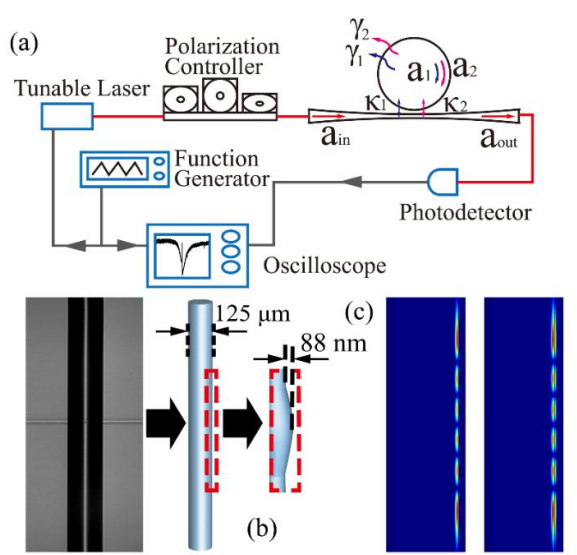

Fig. 1. (a) Illustration of experimental setup. (b) Microscope image of a typical QCMR-microfiber coupling system. (c) Mode field distributions of different radial order modes: $n=1$ and $n=2$ WGMs calculated by COMSOL.
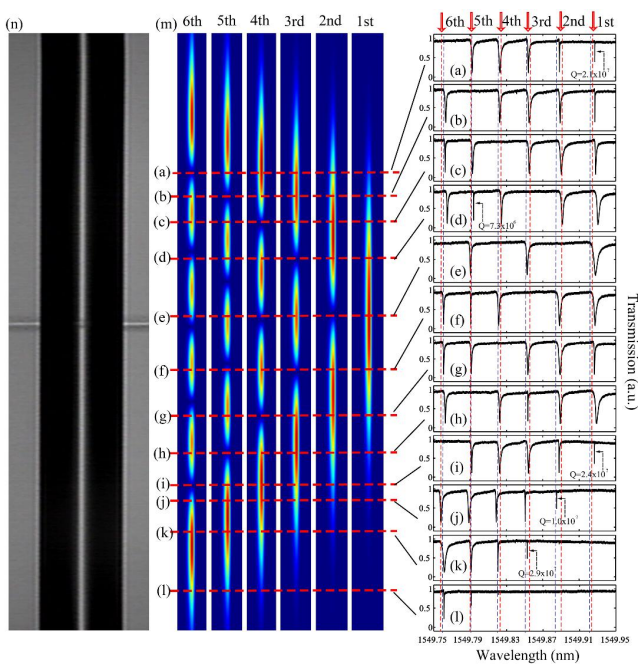

Fig. 2. (a)-(1) Transmission spectra when excited at different axial positions. (m) Light field distributions for the six lowest order axial modes.

(n) Photograph of a typical QCMR.

In order to fabricate a high Q QCMR, we exploited a simple method by using the fiber fusion splicer [16, 17]. First, an ordinary stripped fiber with diameter of $125 \mu \mathrm{m}$ was clamped on the two V-grooves of a commercial fusion splicer (Fujikura FSM-40S); Next, multiple arcs with duration time below one second were applied to soften the fiber locally. During this process, the silica material is heated and expanded, leading to a nanoscale 
radius variation as well as a tiny refractive index (RI) change. The overall effect is manifested as a modification in effective fiber radius $\Delta r_{\text {eff }}$ [18]. The photograph of a typical QCMR, an extremely shallow bottle, with $\sim 400 \mu \mathrm{m}$ in axial direction and only $\sim 88 \mathrm{~nm}$ in radius variation, as shown in Figure. $1 \mathrm{~b}$. Once the resonator is fabricated we glued it on a three-dimensional positioner with $1 \mu \mathrm{m}$ precision. Figure 1 illustrates our experimental setup. A microfiber was used to couple light from a tunable laser (New Focus, TLB-6728) into and out of the QCMR. It is worth noting that the coupling efficiency was adjusted by vertically and horizontally moving the microcavity and, during the whole process, the resonator is always in touch with the microfiber. This is different from the adjusting method used in a microsphere or a microtoroid. A polarization controller was exploited to control the polarization state of input light, and the output light is monitored by a $150 \mathrm{MHz}$ bandwidth InGaAs detector (Thorlabs PDA 10CF-EC) and an OSC.

We next demonstrate selective excitation of high $Q$ localized axial modes in this QCMR-microfiber coupling system. Figures 2(a)-(1) show that, by vertically moving the resonator along its axis, the axial modes disappear and reappear periodically without changing their resonant frequency. Specifically, all of the even order axial WGMs disappear, only remain the first, the third, and the fifth order axial modes, demonstrating the symmetry along the axial direction. As we all know, mode selection and the strength of mode excitation rely on phase matching condition as well as the field overlap between evanescent field of the QCMR and microfiber. This interesting phenomenon is due to the periodical change of spatial overlap between the resonator field and the microfiber field. Figure $2(\mathrm{~m})$ plots the simulated light field distributions for the $1^{\text {st }}$ to the $6^{\text {th }}$ order axial modes of the QCMR, which agrees well with the experimental results. Moreover, the coupling efficiency and Q factor vary when we shifted the resonator, which holds interesting potential in microlasers and high performance sensors. Besides that, during the tuning process, the QCMR is always in contact with microfiber, showing a robust coupling feature. Thanks to the extremely shallow parabolic bulge, our QCMR exhibits highly nondegenerated localized axial modes that are well-separated along its axis, providing good mode-selection capability.

\section{Stable controlled Fano resonances in a QCMR platform}

In this section, we first present the theoretical model of controllable EIT/Fano resonances. And then, by vertically or horizontally moving the resonator, two kinds of stable controlled Fano/EIT lineshapes originated from different mechanisms can be achieved and can work on the same mode. Engineering the on/off contrast ratio of the EIT/Fano lineshapes is also accomplished by scanning the microfiber.

\subsection{Theoretical model}

To generate EIT/Fano resonances in a single QCMR, a high Q mode and a low Q mode should be excited simultaneously in a tiny frequency range. The cavity field amplitudes $a_{1}$ and $a_{2}$ can be expressed by coupled-mode equations (1) [10]:

$$
\begin{aligned}
& \frac{d a_{1}}{d t}=-i \Delta \omega_{1} a_{1}-\frac{\gamma_{1}+\kappa_{1}}{2} a_{1}-g a_{2}-\sqrt{\kappa_{1}} a_{i n} \cos \varphi \\
& \frac{d a_{2}}{d t}=-i \Delta \omega_{2} a_{2}-\frac{\gamma_{2}+\kappa_{2}}{2} a_{2}-g a_{1}-\sqrt{\kappa_{2}} a_{i n} \cos (\varphi-\theta),
\end{aligned}
$$

where $\Delta \omega_{1(2)}=\omega-\omega_{1(2)}$ indicates the resonant detuning between resonator field $a_{1(2)}$ and input light $a_{\text {in }}, \gamma_{1(2)}$ and $\kappa_{1(2)}$ are the intrinsic and coupling loss of WGMs 1(2), respectively. 
$g=\sqrt{\kappa_{1} \kappa_{2}} \cos \varphi / 2$ denotes to the coupling strength between the two cavity modes. $\theta$ and $\varphi$ refer to the polarization angles for $a_{2}$ and $a_{\text {in }}$ relative to $a_{1}$. In the stationary state, by setting $d a_{1(2)} / d t=0$, the transmission amplitude can be written as equation (2):

$$
T=\left|\frac{a_{\text {out }}}{a_{\text {in }}}\right|^{2}=\frac{\left|a_{\text {in }} \cos \theta+\sqrt{\kappa_{1}} a_{1}+\sqrt{\kappa_{2}} a_{2} \cos \varphi\right|^{2}+\left|a_{\text {in }} \sin \theta+\sqrt{\kappa_{2}} a_{2} \sin \varphi\right|^{2}}{\left|a_{\text {in }}\right|^{2}}
$$

From above equation, we discover that, the lineshapes of transmission spectrum can be tuned by adjusting $\kappa_{1(2)}, \gamma_{1(2)}, \varphi, \theta$, and resonant detuning $\Delta \omega_{21}=\omega_{2}-\omega_{1}$. Therefore, one can engineer the EIT/Fano lineshapes through modifying the coupling property, intrinsic feature, polarization degree, or detuning in the platform.

\subsection{Experimental results and discussion}

Fano/EIT resonances can be obtained when modes with closely spaced frequencies are excited and interact with each other. Besides, the quality factor of the high Q mode must be two orders of magnitude larger than that of the low Q mode. In a QCMR, the supported modes are clean and regular when the axial mode order is relatively low (usually below seven), while for higher order axial modes, higher radial order modes $(n \geq 2)$ can also be found. On the one hand, the free spectral range (FSR) of $n=2$ mode is slightly larger than the FSR of $n=1$ mode, leading to the overlap of different radial order WGMs. On the other hand, the existence of the microfiber alters the resonant frequency and the coupling condition of these two modes and, the influence on the fundamental mode is larger [12]. Consequently, the QCMR-based system allows us to study Fano and EIT changing process and the conversion between them by engineering the system through coupling strength or resonant detuning between the two selected modes.

In our experiment, we holded the microfiber in contact with the QCMR, about $100 \mu \mathrm{m}$ away from its equator, swept the input laser and found two modes with relatively large quality factor difference, as shown in figure 3(a). In our case, the low Q mode (mode 2) is the fundamental radial mode, and the high Q mode (mode 1) is the high-order radial mode, but this does not hold for all cases. When gradually moving the QCMR along z-axis, we observed that, mode 1 scans across mode 2 and manifests as an EIT lineshape. During the tuning process, the lineshape of EIT changes (figures 3(b)-(d)), and converts into Fano resonances (figures 3(e)-(f)). We found that, the spectra exhibited various on/off contrast $D=[0.84,0.71,0.39,0.26,0.30]$ and different transparency window linewidth $\Delta \omega_{1}=$ [53.94, 18.98, 8.214, 7.87, 5.66] MHz. The EIT/Fano resonances form as the destructive interference of two pathway related to two excited modes in a single QCMR. With different coupling point, the coupling strength $\kappa_{1(2)}$ and mode detuning $\Delta \omega_{21}$ are steered, leading to evolution of Fano/EIT lineshapes. Notice that Fano resonance forms when both of the two modes are in the overcoupled region $\left(\kappa_{1}>\gamma_{1}\right.$ and $\left.\kappa_{2}>\gamma_{2}\right)$, while EIT lineshape generates when the low Q mode is undercoupled $\left(\kappa_{2}<\gamma_{2}\right)$ and the high Q mode is overcoupled $\left(\kappa_{1}>\gamma_{1}\right)$ [10]. When we further decreased the $z$ position, the two WGMs did not overlap and manifest as two independent Lorentz lineshapes.

In the tuning range about $400 \mu \mathrm{m}$ long, the spectrum may manifest as EIT-like, Fanolike, or two independent Lorentz lineshapes periodically and sequentially depending on the position of microfiber. It is worth noting that the microfiber is always in touch with the QCMR, which makes the system is insensitive to external perturbations such as air flow and slight mechanical vibration, leading to a robust engineering process which is different from previously reported coupling gap tuning method [10,12]. Thanks to the highly nondegenerated localized axial modes manifested by our QCMR, the WGMs are well- 
separated along its axis, providing good mode-selection capability as well as a fine, stable tuning.

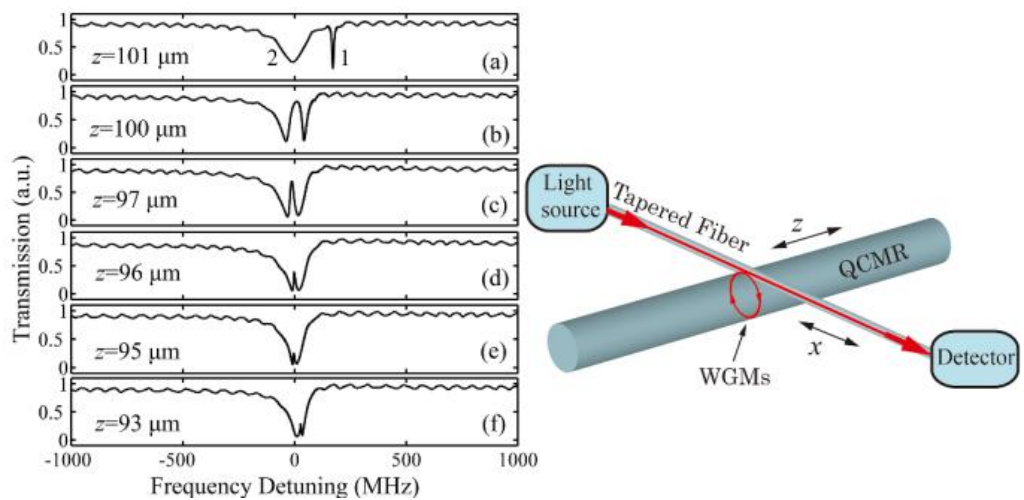

Fig. 3. Experimental transmission spectra with different $z$ position. From top to bottom, the tapered fiber was located at $101 \mu \mathrm{m}, 100 \mu \mathrm{m}, 97 \mu \mathrm{m}, 96 \mu \mathrm{m}, 95 \mu \mathrm{m}$, and $93 \mu \mathrm{m}$ away from the equator. The inset describes the engineering process.
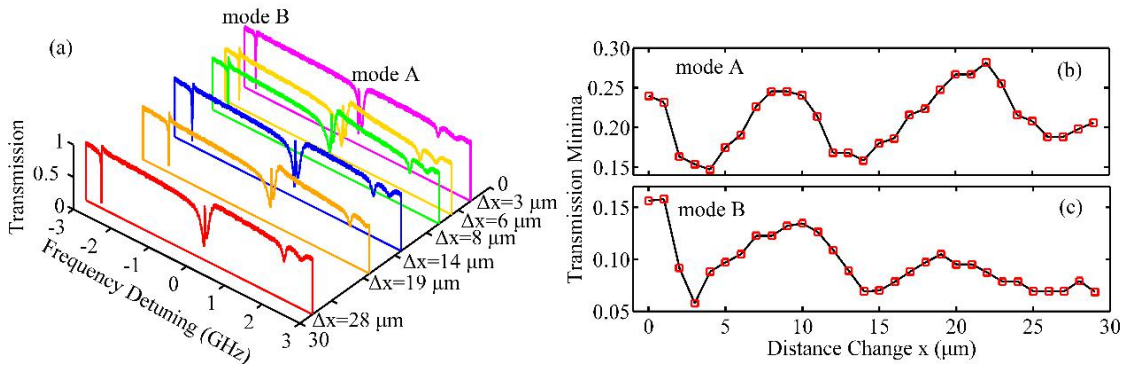

Fig. 4. (a) Experimental spectra evolution with various horizontal positions: $\Delta x=3 \mu \mathrm{m}, 6 \mu \mathrm{m}, 8 \mu \mathrm{m}$, $14 \mu \mathrm{m}, 19 \mu \mathrm{m}$, and $28 \mu \mathrm{m}$. Transmission minima of (b) mode A and (c) mode B for different distance change $\Delta x$.

We finally investigate another kind of periodically evolved Fano resonances by horizontally moving the QCMR. With different $\Delta x$ position, the transmission spectra converted from symmetric Lorentz $(\Delta x=3 \mu \mathrm{m})$ to asymmetric Fano-like lineshape $(\Delta x=6,8$ $\mu \mathrm{m})$ and then changed back to the symmetric lineshape $(\Delta x=14 \mu \mathrm{m})$, and so forth $(\Delta x=19$, $28 \mu \mathrm{m}$ for Fano-like lineshape), as depicted in figure 4(a). In order to confirm the periodical variation feature of Fano resonance, figure 4(b)-(c) give the transmission minima of the spectra versus distance change $x$ for mode A and mode B respectively. We acquire the data every one micrometer within a displacement range of $\sim 30 \mu \mathrm{m}$. Apparently, the Fano resonance changes with a period of $\sim 15 \mu \mathrm{m}$, and the phenomenon does not change if scanning backward.

This dynamically evolved Fano resonance results from multimode dispersion and mode coupling in the microfiber. Notice that for mode A, both the periodical Fano and the EITlike behavior are exhibited, that is to say, two different mechanisms that induce Fano can work on the same mode without disturbing the other. We explain this phenomenon using the modified multimode waveguide coupled WGMs model, the schematic diagram can be obtained in [9]. The Fano resonance can be expressed as equation (3) and (4):

$$
\begin{gathered}
T=\left|\frac{E_{t}}{E_{0}}\right|^{2}=\frac{|\bar{t}|^{2}\left(\omega-\omega_{c}\right)^{2}+C_{1}+C_{2}\left(\omega-\omega_{c}\right)}{\left(\omega-\omega_{c}\right)^{2}+\left(1-\alpha_{c} t_{c}\right)^{2} v_{c}^{2}} \\
C_{1}=\left|\left(1-\alpha_{c} t_{c}\right) \bar{t}+\alpha_{c}\left(\bar{p}_{1} \bar{q}_{1}-\bar{p}_{2} \bar{q}_{2}\right)\right|^{2} v_{c}^{2}, C_{2}=2 \alpha_{c} \operatorname{Im}\left[\bar{t}\left(\bar{p}_{1} \bar{q}_{1}-\bar{p}_{2} \bar{q}_{2}\right)^{*}\right] v_{c} .
\end{gathered}
$$


where $E_{0}$ and $E_{\mathrm{t}}$ indicate the input and output light in the microfiber. $\bar{t}=\sum_{j} t_{j}, \bar{p}_{1(2)}=\sum_{j} p_{1(2) j}$, and $\bar{q}_{1(2)}=\sum_{j} q_{1(2) j}$ represent direct transmittances indices, coupling indices from single microfiber modes to cavity modes ( 1 or 2 ) and the coupling indices from the cavity mode ( 1 or 2) to microfiber field. We assume $\alpha_{c 1} \approx \alpha_{c 2}=\alpha_{c}, \omega_{c 1} \approx \omega_{c 2}=\omega_{c}, v_{c 1} \approx v_{c 2}=v_{c}$, and $t_{c 1} \approx t_{c 2}=t_{c}$ for simplicity. $\alpha_{c}$ and $t_{c}$ are the round-trip coefficient and the transmission coefficient. $w_{c}$ and $v_{c}$ refer to the resonance frequency and the FSR of a WGM 1(2). Equation (3)-(4) show that the first two terms represent a symmetric Lorentzian dip with an EIT peak, the last term manifests as an asymmetric Fano lineshape. The interaction of microfiber modes leads to $C_{2} \neq 0$, generating the Fano resonance.

In addition, the phase shifted coupling coefficients $p_{1(2) j}$ and $q_{1(2) j}$ lead to $\left.\bar{t}\left(\bar{p}_{1} \bar{q}_{1}-\bar{p}_{2} \bar{q}_{2}\right)^{*}\right|_{x}=\sum_{j, k, l}\left\{\left.t_{j}\left(p_{1 k} q_{1 l}-p_{2 k} q_{2 l}\right)^{*}\right|_{x=0} \exp \left(-i\left(\beta_{k}-\beta_{l}\right) x\right)\right\}$. Assume that two modes existing in the microfiber, the Fano resonance evolves periodically with a period of $2 \pi\left|\beta_{1}-\beta_{2}\right|^{-1}$, with $\beta_{j}(j=1,2, \ldots)$ indicate the propagation constant of modes in microfiber. Besides that, a $2.4 \mu \mathrm{m}$ microfiber can support $\mathrm{HE}_{11}, \mathrm{TE}_{01}, \mathrm{HE}_{21}, \mathrm{TM}_{01}, \mathrm{EH}_{11}$, and $\mathrm{HE}_{31}$ modes with propagation constant of $5.5496 \mu \mathrm{m}^{-1}, 5.1883 \mu \mathrm{m}^{-1}, 5.1070 \mu \mathrm{m}^{-1}, 5.0817 \mu \mathrm{m}^{-1}$, 4.6209 $\mu \mathrm{m}^{-1}$, and $4.4785 \mu \mathrm{m}^{-1}$. We can obtain the periods for mode pairs $\mathrm{HE}_{11} / \mathrm{TE}_{01}$, $\mathrm{HE}_{11} / \mathrm{HE}_{21}, \mathrm{HE}_{11} / \mathrm{TM}_{01}, \mathrm{HE}_{11} / \mathrm{EH}_{11}$, and $\mathrm{HE}_{11} / \mathrm{HE}_{31}$ as $17.4 \mu \mathrm{m}, 14.2 \mu \mathrm{m}, 13.4 \mu \mathrm{m}, 6.7 \mu \mathrm{m}$, and $5.9 \mu \mathrm{m}$, respectively. Considering the experimental Fano period of $\sim 11 \mu \mathrm{m}$, locating between $17.4 \mu \mathrm{m}$ and $14.2 \mu \mathrm{m}$, we believe that the periodically changed Fano resonances observed in figure 4 are due to the coupling between $\mathrm{HE}_{11}, \mathrm{TM}_{01}, \mathrm{HE}_{21}$ microfiber modes and the cavity modes.

Compared with previously reported Fano resonances in a single microsphere, the phenomenon here is more obvious and stable. Remarkably, two different mechanisms that create the Fano/EIT resonances work on the same WGM without disturbing each other.

\section{Conclusion}

In summary, we have investigated the stable controlling and engineering of Fano and EITlike resonances in a robust QCMR photonic system, by vertically or horizontally moving the resonator. The two kinds of phenomena originate from taper-mediated interference between the two whispering gallery modes and the multimode coupling in the fiber, respectively. Importantly, they can work on the same mode simultaneously. The photonic platform, demonstrated in this work, provides a robust platform for accessing, controlling, and engineering the Fano/EIT resonances, which may hold unique potential in optical switching, slow light, and sensitivity-enhanced sensing applications.

\section{Acknowledgments}

This work is supported by the National Natural Science Foundation of China (NSFC) (grant 61775209, 61275011).

\section{References}

1. Fano U Effects of configuration interaction on intensities and phase shifts Phys. Rev. 1241866 (1961)

2. Chao C-Y and Guo L J Biochemical sensors based on polymer microrings with sharp asymmetrical resonance Appl. Phys. Lett. 83 1527-9 (2003) 
3. Liu C, Dutton Z, Behroozi $\mathrm{C} \mathrm{H}$ and Hau L V Observation of coherent optical information storage in an atomic medium using halted light pulses Nature 409 490-3 (2001)

4. Phillips D, Fleischhauer A, Mair A, Walsworth R and Lukin M D Storage of light in atomic vapor Phys. Rev. Lett. 86783 (2001)

5. Hau L V, Harris S E, Dutton Z and Behroozi C H Light speed reduction to 17 metres per second in an ultracold atomic gas Nature 397 594-8 (1999)

6. Tomita M, Totsuka K, Hanamura R and Matsumoto T Tunable Fano interference effect in coupled-microsphere resonator-induced transparency J. Opt. Soc. Am. B 26 813-8 (2009)

7. Ghulinyan M, Manzano F R, Prtljaga N, Bernard M, Pavesi L, Pucker G and Carusotto I Intermode reactive coupling induced by waveguide-resonator interaction Phys. Rev. A 90053811 (2014)

8. Peng B, Özdemir Ş K, Chen W, Nori F and Yang L What is and what is not electromagnetically induced transparency in whispering-gallery microcavities Nat. Commun. 5 (2014)

9. Chiba A, Fujiwara H, Hotta J-i, Takeuchi S and Sasaki K Fano resonance in a multimode tapered fiber coupled with a microspherical cavity Appl. Phys. Lett. 86 261106 (2005)

10. Li B-B, Xiao Y-F, Zou C-L, Liu Y-C, Jiang X-F, Chen Y-L, Li Y and Gong Q Experimental observation of Fano resonance in a single whispering-gallery microresonator Appl. Phys. Lett. 98021116 (2011)

11. Lei F, Peng B, Özdemir Ş K, Long G L and Yang L Dynamic Fano-like resonances in erbium-doped whispering-gallery-mode microresonators Appl. Phys. Lett. 105101112 (2014)

12. Dong C-H, Zou C-L, Xiao Y-F, Cui J-M, Han Z-F and Guo G-C Modified transmission spectrum induced by two-mode interference in a single silica microsphere J. Phys. B 42215401 (2009)

13. Wang Y, Zhang K, Zhou S, Wu Y-H, Chi M-B and Hao P Coupled-mode induced transparency in a bottle whispering-gallery-mode resonator Opt. Lett. 41 1825-8 (2016)

14. Yang Y, Saurabh S, Ward J and Chormaic S N Coupled-mode-induced transparency in aerostatically tuned microbubble whispering-gallery resonators Opt. Lett. 40 1834-7 (2015)

15. Xiao Y-F, He L, Zhu J and Yang L Electromagnetically induced transparency-like effect in a single polydimethylsiloxane-coated silica microtoroid Appl. Phys. Lett. 94 231115 (2009)

16. Dong $\mathrm{Y}$, Jin $\mathrm{X}$ and Wang $\mathrm{K}$ Packaged and robust microcavity device based on a microcylinder-taper coupling system Appl. Opt. 54 4016-22 (2015)

17. Jin $\mathrm{X}$, Dong $\mathrm{Y}$ and Wang $\mathrm{K}$ Selective excitation of axial modes in a high-Q microcylindrical resonator for controlled and robust coupling Appl. Opt. 54 8100-7 (2015)

18. Sumetsky M, DiGiovanni D, Dulashko Y, Fini J, Liu X, Monberg E and Taunay T Surface nanoscale axial photonics: robust fabrication of high-quality-factor microresonators Opt. Lett. 36 4824-6 (2011) 